Supplementary Informations

\title{
A Facile Procedure for Controlling Monomer Sequence Distribution in Radical Chain Polymerizations
}

\author{
Sebastian Pfeifer and Jean-François Lutz* \\ Research Group Nanotechnology for Life Science, Fraunhofer Institute for Applied \\ Polymer Research, Geiselbergstrasse 69, Potsdam-Golm 14476, Germany \\ E-Mail: lutz@iap.fhg.de
}

\section{A - Experimental part}

Chemicals. $N$-Propylmaleimide (PMI) (Aldrich, 95\%), $N$-benzylmaleimide (BzMI) (Aldrich, 99\%), $\mathrm{N}$-methylmaleimide (MMI) (Aldrich, 97\%), $\mathrm{N}$-phenylmaleimide (Fluka, 98\%), $\mathrm{N}$ methoxycarbonyl-maleimide (Fluka, 97\%), $N$-(4-azobenzene) maleimide (Aldrich, 97\%), copper(I) bromide (Acros, 98\%), styrene (Aldrich, 99\%), (1-bromoethyl) benzene (Acros, 97\%) and 4,4'-dinonyl-2,2'-bipyridine (dNbipy) (Aldrich, 97\%) were used as received. $\mathrm{N}$ [3,5-Bis(trifluoromethyl)phenyl] maleimide (TFMPMI) was synthesized according to published procedures. ${ }^{1}$

Sequential atom transfer radical copolymerization of Styrene, PMI, BzMI, MMI and TFMPMI. (1) Preparation of the initial reaction: $N$-Propylmaleimide (112.8 mg, 0.81 mmol, 3 Eq.), copper bromide (38.8 mg, 0.27 mmol, 1 Eq.) and 4,4'-dinonyl-2,2'-bipyridine (220.9 mg, 0.54 mmol, 2 Eq.) were added into a Schlenk tube. The tube was sealed with a septum and subsequently purged with dry argon for a few minutes. Then, $3.1 \mathrm{~mL}$ of thoroughly degassed styrene ( $2.8 \mathrm{~g}, 27 \mathrm{mmol}, 100 \mathrm{Eq}$.) were added with a degassed syringe. The mixture turned dark brown, indicating complexation of $\mathrm{Cu}(\mathrm{I}) \mathrm{Br}$ and dNBipy. Lastly, (1bromoethyl) benzene (50 mg, $0.27 \mathrm{mmol}, 1 \mathrm{Eq}$.) was added with a precision syringe. The mixture was heated at $110^{\circ} \mathrm{C}$ in an oil bath for several hours. (2) Controlled Addition of BzMI: After 1 hour of polymerization, a thoroughly degassed solution of $N$-benzylmaleimide (151 mg, $0.81 \mathrm{mmol}, 3 \mathrm{Eq.)}$ in $0.8 \mathrm{~mL}$ of toluene was added with a degassed syringe. Immediately after addition, a polymerization sample was taken with a degassed syringe and analyzed by ${ }^{1} \mathrm{H}$ NMR and SEC. (3) Controlled Addition of MMI: After 2 hours and 45 minutes of polymerization, a thoroughly degassed solution of $N$-methylmaleimide $(90 \mathrm{mg}$, $0.81 \mathrm{mmol}, 3 \mathrm{Eq}$.) in $0.8 \mathrm{~mL}$ of toluene was added with a degassed syringe. Immediately after addition, a polymerization sample was taken with a degassed syringe and analyzed by ${ }^{1} \mathrm{H}$ 
NMR and SEC. (4) Controlled Addition of TFMPMI: After 6 hours of polymerization, a thoroughly degassed solution of $N$-[3,5-Bis(trifluoromethyl)phenyl] maleimide (250 mg, 0.81 mmol, 3 Eq.) in $0.8 \mathrm{~mL}$ of toluene was added with a degassed syringe. (4) Final purification: After 21 hours of polymerization, the experiment was stopped by opening the flask and exposing the catalyst to air. Subsequently, the copolymer was purified by selective precipitation in methanol. The purified polymer appeared as a white powder.

Measurements and analysis. Size Exclusion Chromatography, SEC in THF: Molecular weights and molecular weight distributions were determined by SEC performed at $25^{\circ} \mathrm{C}$ in tetrahydrofuran (THF) as eluent (flow rate: $1 \mathrm{~mL} \cdot \mathrm{mn}^{-1}$ ), using three SDV columns (Polymer Standards Service $\mathrm{GmbH}$ ) with a particle size of $5 \mu$ and a porosity of $10^{3}, 10^{5}$ and $10^{6} \AA$ (the porosity values do not correspond to real pore diameters but to manufacturer standards). The detection was performed with a RI- (Shodex RI-71) and a UV-Detector (TSP UV 1000; 260 $\mathrm{nm}$ ). For calibration, linear polystyrene standards (Polymer Standards Service GmbH) were used. Nuclear Magnetic Resonance. ${ }^{1} \mathrm{H}$ NMR Spectra were recorded in $\mathrm{CDCl}_{3}$ on a Bruker DPX-400 operating at 400.1 MHz. Figure S1 shows a typical ${ }^{1} \mathrm{H}$ NMR spectrum recorded during a sequential radical copolymerization of styrene and various $N$-substituted-maleimides.

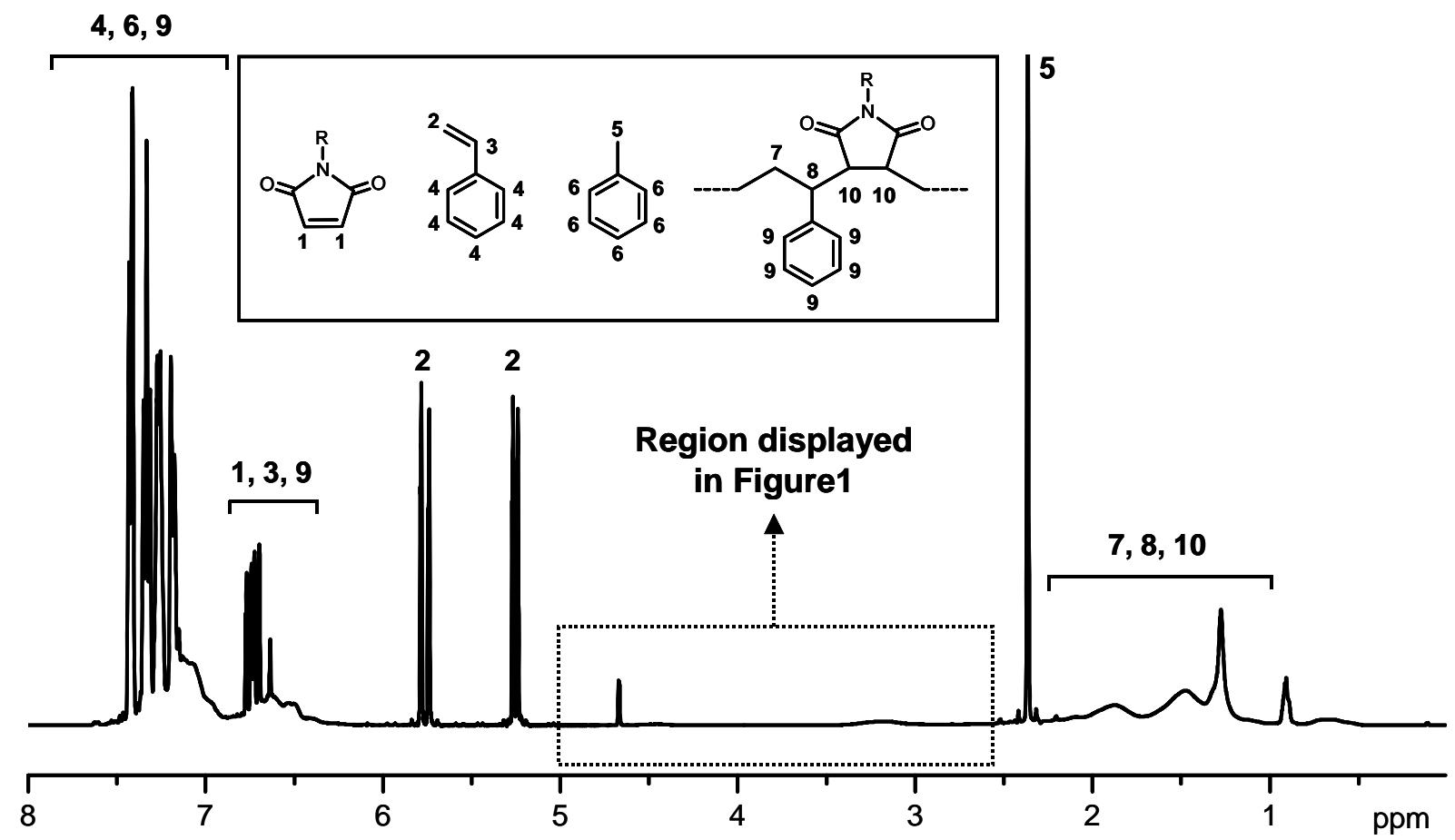

Figure S1. Example of ${ }^{1} \mathrm{H}$ NMR spectrum recorded for the sequential ATRP of styrene and various $\mathrm{N}$-substituted-maleimides (this particular spectrum corresponds to Figure $1 \mathrm{~B}$ in the main manuscript). 
Styrene conversion ( $\left.\operatorname{conv}_{\mathrm{S}}\right)$ was calculated from the ${ }^{1} \mathrm{H}$ NMR spectra of the raw experimental samples by comparing the integration of one vinyl protons of remaining styrene at $5.8 \mathrm{ppm}$ to the integration of the region 6.90-6.05 ppm, which contains 2 aromatic protons of the formed copolymer, 1 methine proton of the remaining styrene and at some stages of the reaction 2 protons belonging to the added $\mathrm{N}$-substituted-maleimides. The conversions of $\mathrm{N}$ propylmaleimide, $N$-benzylmaleimide and $N$-methylmaleimide were estimated by ${ }^{1} \mathrm{H}$ NMR. For PMI, the evolution of the triplet at $3.48 \mathrm{ppm}$ (2 methylene protons in alpha of the maleimide function) was monitored. For BzMI, the evolution of the singlet at $4.67 \mathrm{ppm}$ (2 methylene protons of the benzyl group) was studied. For MMI, the evolution of the singlet at 3 ppm (3 methyl protons) was monitored. ${ }^{19} \mathrm{~F}$ NMR Spectra were recorded in $\mathrm{CDCl}_{3}$ with a $300 \mathrm{MHz}$ Bruker Avance instrument.

\section{B - Additional copolymerization data.}

\section{Atom transfer radical copolymerization of styrene with various functional maleimides.}

The goal of the present communication is to highlight that the sequential atom transfer radical copolymerization of styrene and $N$-substituted maleimides could be used a general platform for preparing polymers with a programmed distribution of functional groups. In this context, it was first important to demonstrate that this experimental strategy is versatile (i.e. that various $\mathrm{N}$-substituted maleimides can be used in ATRP). Maleimide rings (MI) may be substituted on their $N$-site by various functional groups. Such $N$-substituted maleimides are usually obtained via the reaction of maleic anhydride with a substituted primary amine, followed by a condensation step. Alternatively, they can be prepared via the Mitsunobu reaction of substituted alcohols with maleimide. ${ }^{2}$ Thus, a plethora of $N$-substituted maleimides have been already reported in the literature. Many of them have been copolymerized with styrene via conventional free-radical polymerization. ${ }^{3}$ However, only a few research groups studied the atom transfer radical copolymerization of styrene with $\mathrm{N}$-substituted maleimides. ${ }^{4}, 5$ Nevertheless, it was evidenced that ATRP allows an efficient control of the alternating copolymerization of styrene with either $N$-phenylmaleimide, $N$-(2-acetoxyethyl) maleimide, $N$-cyclohexyl maleimide or $N$-butyl maleimide. To demonstrate further the versatility of ATRP for preparing P(S-co-MI) copolymers, the bulk ATRP of styrene was studied at $110^{\circ} \mathrm{C}$ in the presence of six model comonomers (Table S1). $\mathrm{N}$-Methylmaleimide (1) and $\mathrm{N}$ propylmaleimide (2) were studied as models for $\mathrm{N}$-alkyl maleimides. Additionally, two aromatic models $N$-benzylmaleimide (3) and $N$-phenylmaleimide (4) were investigated. Maleimides bearing more polar substituents (i.e. neutral or charged hydrophilic moieties) may also be of interest. However, free carboxylic acids, primary amines or secondary amines are 
usually problematic in ATRP since these particular functions interact with transition metal catalysts. ${ }^{6}$ Thus, protected structures (e.g. tert-butyl or benzyl esters) are usually used in ATRP. For instance, $N$-methoxycarbonyl-maleimide (5) was used a general model for maleimide esters. The sequential copolymerization of styrene and maleimides could be also used to incorporate specific substituents (reactive, fluorescent or stimuli-responsive moieties) at precise locations in well-defined copolymer chains. As an example, the light-responsive monomer $N$-(4-azobenzene) maleimide (7) was studied.

Table S1. Properties of well-defined copolymers P(S-co-MI) prepared by ATRP. ${ }^{a}$

\begin{tabular}{cccccc}
\hline $\mathbf{M I}^{\mathbf{b}}$ & $\mathbf{S} / \mathbf{M I} / \mathbf{P E B} / \mathbf{C u B r} / \mathbf{d N b i p y}$ & $\mathbf{t i m e ~}^{\mathbf{c}}$ & $\boldsymbol{M}_{\mathbf{n}}$ & $\boldsymbol{M}_{\mathbf{n}}$ th & $\boldsymbol{M}_{\mathbf{w}} / \boldsymbol{M}_{\mathbf{n}}$ \\
\hline $\mathbf{1}$ & $100 / 3 / 1 / 1 / 2$ & 6 & 8900 & 9700 & 1.25 \\
$\mathbf{2}$ & $100 / 3 / 1 / 1 / 2$ & 6 & 9600 & 9900 & 1.21 \\
$\mathbf{3}$ & $100 / 3 / 1 / 1 / 2$ & 6 & 10100 & 9700 & 1.21 \\
$\mathbf{4}$ & $100 / 3 / 1 / 1 / 2$ & 4 & 7500 & 7900 & 1.31 \\
$\mathbf{5}$ & $100 / 3 / 1 / 1 / 2$ & 7 & 5400 & 4500 & 1.28 \\
$\mathbf{7}$ & $100 / 3 / 1 / 1 / 2$ & 6 & 6300 & 6030 & 1.35 \\
\hline
\end{tabular}

${ }^{a}$ All copolymerizations were performed in bulk at $110^{\circ} \mathrm{C} .{ }^{\mathrm{b}} \mathrm{MI}$ is an acronym for $\mathrm{N}$ substituted-maleimide. ${ }^{\mathrm{c}}$ PEB stands for the initiator 1-phenyl ethyl bromide (a.k.a. (1bromoethyl) benzene).

The data shown in Table S1 confirm that ATRP is an efficient method for preparing welldefined P(S-Co-MI) copolymers. All bulk polymerizations reached high conversions within a few hours of reaction, which is in good agreement with previously reported kinetic studies. ${ }^{7}$ Moreover, ${ }^{1} \mathrm{H}$ NMR measurements indicated that the $N$-substituted maleimides are almost completely consumed (conversion $>99.9 \%$ ) in all these copolymerizations (see paragraph below for detailed kinetics of copolymerization). All the formed copolymers were found to be well-defined (experimental molecular weights matched well expected theoretical values and polydispersity indexes were in most cases relatively low).

Model kinetics of copolymerization. Most of the $N$-substituted maleimides copolymerize with styrene in an alternating fashion. ${ }^{8}$ However, this behavior depends on the mole fraction of maleimide in the comonomer feed. For very low maleimide mole fractions, the copolymerization is usually not strictly alternating, although the maleimide is generally consumed much faster than styrene. The sequential concept introduced in the present 
communication relies on this preferential maleimide consumption. Thus, it was very important to study this aspect in details. The kinetics of the atom transfer radical copolymerization of styrene with either $\mathrm{N}$-propylmaleimide, $\mathrm{N}$-benzylmaleimide or $\mathrm{N}$ methylmaleimide were studied by ${ }^{1} \mathrm{H}$ NMR. In all copolymerizations, very low mole fractions of maleimides (i.e. 2.9\%) were used in the comonomer feed. Figure S2 shows the semilogarithmic plots of monomer conversions as a function of time for these three radical copolymerizations.
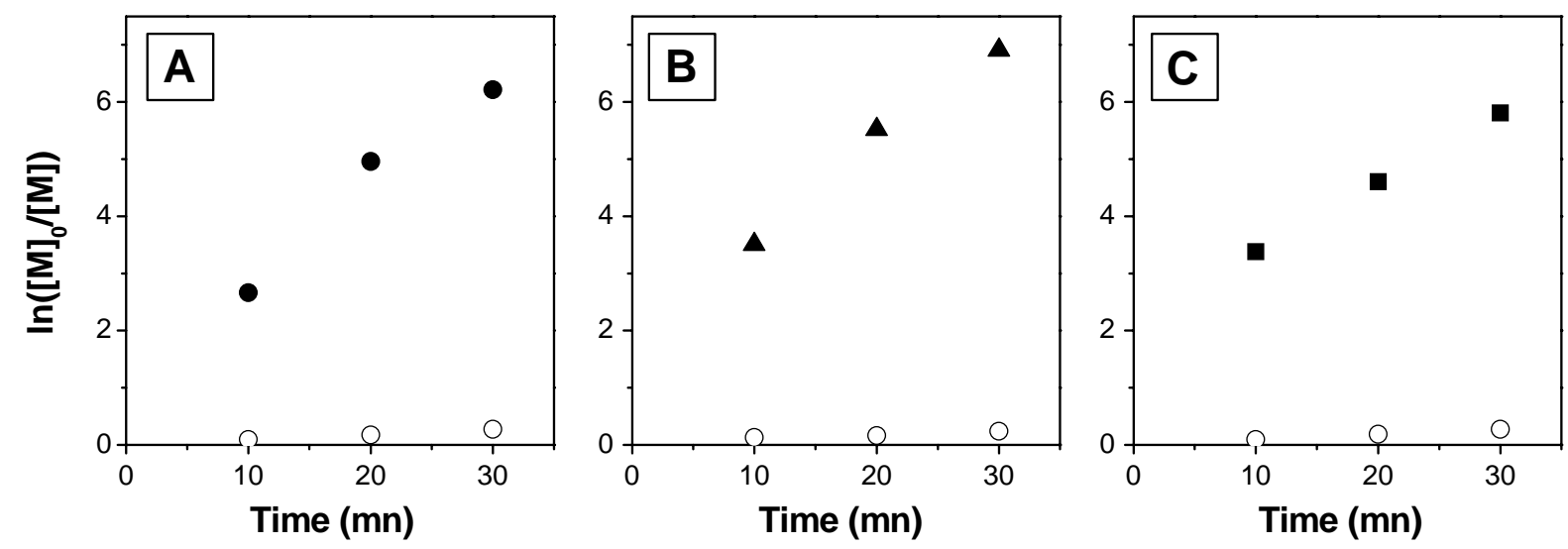

Figure S2. Semi logarithmic plots of monomer conversion versus time recorded for the bulk atom transfer radical copolymerization of styrene (empty circles in all graphs) with either (A) $\mathrm{N}$-propylmaleimide (full circles), (B) $\mathrm{N}$-benzylmaleimide (full triangles) or (C) $\mathrm{N}$ methylmaleimide (full squares). The experimental conditions were in all cases: $110^{\circ} \mathrm{C}$; $\mathrm{S} / \mathrm{MI} / \mathrm{PEB} / \mathrm{CuBr} / \mathrm{dNbipy}=100 / 3 / 1 / 1 / 2$.

In all copolymerizations, it appears that, although present in very low amounts, the $\mathrm{N}$ substituted maleimides are effectively consumed much faster than styrene. After 15 minutes of polymerization, the conversion of the maleimides was found in all cases to be above $99 \%$, whereas the conversion of styrene was only about $10 \%$. Thus the formed copolymers chains are composed of a first short block (DP 12) containing $75 \%$ of styrene and $25 \%$ of maleimides (i.e. two maleimide moieties are separated by three styrene units in average) and subsequently of a long block of homopolystyrene. These results are in very good agreement with the data reported by Hawker and coworkers for the nitroxide mediated copolymerization of styrene and maleic anhydride. ${ }^{9}$

Kinetic study of the microstructure of the formed sequential copolymer. Table S2 shows the evolution of the monomer concentrations (obtained from NMR data) during the sequential atom transfer radical copolymerization of styrene, PMI, BzMI, MMI and TFMPMI. Since the studied polymerization is a living process (i.e. all the chains are initiated and grow 
simultaneously), ${ }^{7,} 10$ the monomer sequence distribution of the copolymer may be estimated from these kinetics of polymerization.

Table S2. Evolution of the monomer concentrations (expressed in mole equivalents, as compared to the initial concentration of initiator) and of the overall degree of polymerization $(D P)$ during the copolymerization.

\begin{tabular}{ccccccc}
\hline Step & S & PMI & BzMI & MMI & TFPMI & DP \\
\hline Time 0 & 100 & 3 & - & - & - & 0 \\
Addition 1 & 64 & 0 & 3 & - & - & 39 \\
Addition 2 & 50 & 0 & 0 & 3 & - & 56 \\
Addition 3 & 41 & 0 & 0 & 0 & 3 & 69 \\
End & 35 & 0 & 0 & 0 & 0 & 77 \\
\hline
\end{tabular}

The copolymerization was first started in the presence of 100 Eq. of styrene and 3 Eq. of PMI. Model kinetics evidenced (see previous paragraph) that PMI is fully polymerized within 15 minutes, whereas only $10 \%$ of the styrene is consumed. Thus, a first block $\mathrm{P}(\mathrm{S}-\mathrm{CO}$-PMI) with a degree of polymerization of approximately 10-15 is formed during these early stages of the reaction. This first segment contains in average 3 styrene units between each PMI motif (see previous paragraph). Afterwards, the polymerization was continued for 45 minutes leading to the formation of a polystyrene block containing approximately 25 monomer units (Figure S3). After one hour of polymerization, SEC confirmed that a copolymer with a DP of approximately 35 was formed.

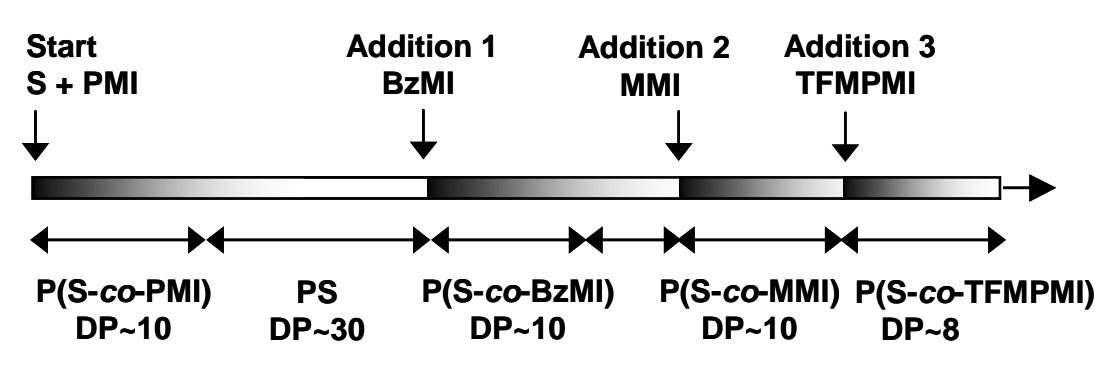

Figure S3. Schematic representation of the microstructure of the formed copolymer.

After the addition of BzMI, the copolymerization with styrene proceeded rapidly (see model kinetics). The tendency toward alternation is much stronger at this stage of the reaction since the amount of remaining styrene is lower. Thus, a third block P(S-co-MMI) is rapidly formed, probably followed by a short polystyrene section of approximately 5-10 units (Scheme S3). The fifth block is composed of styrene and MMI with probably a very high tendency towards 
alternation. The sixth and last block is composed of styrene and TFMPMI. There is most likely no significant homopolystyrene spacer between these last two blocks (Figure S3).

\section{References}

1. El-Guweri, M.; Hendlinger, P.; Laschewsky, A. Macromol. Chem. Phys. 1997, 198, 401-418.

2. Walker, M. A. J. Org. Chem. 1995, 60, 5352-5355.

3. Brandrup, J.; Immergut, E. H.; Grulke, E. A. Ed., Polymer Handbook fourth edition. Wiley-Intersience: Hoboken, 1999; p.

4. $\quad$ Chen, G. Q.; Wu, Z. Q.; Wu, J. R.; Li, Z. C.; Li, F. M. Macromolecules 2000, 33, 232234.

5. Zhao, Y.-L.; Chen, C.-F.; Xi, F. J. Polym. Sci. Part A: Polym. Chem. 2003, 41, 21562165.

6. Matyjaszewski, K.; Xia, J. Chem. Rev. 2001, 101, 2921-2990.

7. Lutz, J.-F.; Matyjaszewski, K. J. Polym. Sci. Part A: Polym. Chem. 2005, 43, 897-910.

8. Rzaev, Z. M. O. Prog. Polym. Sci. 2000, 25, 163.

9. Benoit, D.; Hawker, C. J.; Huang, E. E.; Lin, Z.; Russell, T. P. Macromolecules 2000, 33, 1505-1507.

10. Lutz, J.-F.; Matyjaszewski, K. Macromol. Chem. Phys. 2002, 203, 1385-1395. 